\title{
Burden of intestinal helminths and associated factors three years after initiation of mass drug administration in Arbaminch Zuria district, Southern Ethiopia
}

\author{
Getaneh Alemu ${ }^{1 *}$, Zeleke Aschalew ${ }^{2}$ and Eshetu Zerihun ${ }^{3}$
}

\begin{abstract}
Background: Epidemiological information on the burden of various parasitic infections and associated factors in different localities is indispensable to develop appropriate control and prevention measures as well as to monitor and evaluate existing programs. Hence the aim of the present study was to assess the prevalence and associated factors of intestinal helminths among school age children in Arbaminch Zuria district, Southern Ethiopia.

Methods: A community based cross-sectional study was conducted by recruiting 391 primary school age children. Participants were selected using simple random sampling technique. Pre-tested structured questionnaire was used to collect data about socio-demographic characteristics and perceived factors associated with intestinal parasitosis. Stool microscopic examination was done following standard protocol. Data were analyzed using Statistical Package for Social Science version 22.

Results: Among 391 children participated, 181 (46.3\%; 95\% Cl: 41.9-51.5) were infected with intestinal helminths. The magnitudes of single and double infections were $40.2 \%$ and $6.1 \%$ respectively. Seven helminths were detected among which Ascaris lumbricoides was with the highest frequency (56, 14.3\%) followed by hookworms (55, 14.1\%). Not washing fruits and vegetables before consumption ( $\mathrm{AOR}=2.16$; $95 \% \mathrm{Cl}: 1.10-4.25, p=0.026$ ) and habit of swimming (AOR $=1.17 ; 95 \% \mathrm{Cl}: 1.03-1.95, p=0.045)$ were significantly associated with helminthic infection.

Conclusion: High prevalence of intestinal helminthic infections among school age children demands integration of deworming with water, hygiene and sanitation as well as consistent health education.
\end{abstract}

Keywords: Prevalence, Helminth, School age children

\section{Background}

Globally about two billion people are infected with intestinal helminths mainly in resource-poor settings; children being the most affected population groups [1]. The big three intestinal helminths, commonly named soil transmitted helminths $(\mathrm{STH})$, pose the most common parasitic infections worldwide. About 819 million people are infected with Ascaris lumbricoides, 465 million with Trichuris trichiura, and 439 million with hookworms [2].

\footnotetext{
* Correspondence: getanehmlt@gmail.com

${ }^{1}$ Department of Medical Laboratory Science, College of Medicine and Health Sciences, Bahir Dar University, Bahir Dar, Ethiopia

Full list of author information is available at the end of the article
}

Worldwide 800 million individuals are at risk of intestinal schistosomiasis; about 200 million people $(160$ million live in Sub-Saharan Africa) are estimated to be infected [3]. Ethiopia is one of the Sub-Sahara African countries with heavy burden of intestinal helminths. Soil transmitted helminth infections are distributed throughout the country that 81 million people live in endemic areas, of which, 25.3 million are school-age children (SAC). There are an estimated 38.3 million people (12.3 million are SAC) living in schistosomiasis endemic areas [4].

Intestinal helminths can infect all age groups but the magnitude and morbidity outweighs among primary SAC. Recent data show high burden of intestinal helminths among SAC (Table 1). 
Table 1 Previous studies showing magnitude of intestinal parasites among school age children

\begin{tabular}{|c|c|c|c|c|}
\hline \multicolumn{2}{|c|}{ Study Site (Citation) } & Magnitude of Intestinal helminths & Laboratory Method used & Sample size \\
\hline \multicolumn{2}{|l|}{ Nepal [5] } & $27.67 \%$ & Direct wet mount & 495 \\
\hline \multicolumn{2}{|c|}{ Yemen [6] } & $90 \%$ & Direct wet mount and Formol-ether & 330 \\
\hline \multicolumn{2}{|c|}{ Nigeria [7] } & $63.49 \%$ & Formol-ether & 252 \\
\hline \multicolumn{2}{|c|}{ Sao Tome and Principe [8] } & $64.7 \%$ & Merthiolate-iodine-formaldehyde & 252 \\
\hline \multicolumn{2}{|l|}{ Sudan [9] } & $24.9 \%$ & Kato-katz & 662 \\
\hline \multicolumn{2}{|c|}{ Sudan [10] } & $84 \%$ & Direct wet mount and Formol-ether & 120 \\
\hline \multicolumn{2}{|c|}{ Sudan [11] } & $30 \%$ & Formol-ether & 200 \\
\hline \multicolumn{2}{|c|}{ Kenya [12] } & $44.05 \%$ & Kato-katz & 377 \\
\hline \multirow[t]{8}{*}{ Ethiopia } & Bahir Dar [13] & $65.5 \%$ & Formol-ether & 359 \\
\hline & Zege [14] & $69.1 \%$ & Formol-ether & 408 \\
\hline & Maksegnit and Enfranz [15] & $66.4 \%$ & Kato-katz & 550 \\
\hline & Chencha [16] & $36.8 \%$ & Kato-katz & 408 \\
\hline & Arba Minch zuria [17] & $39.9 \%$ & Direct wet mount and Formol-ether & 858 \\
\hline & Hawassa [18] & $67.9 \%$ & Formol-ether & 374 \\
\hline & Wolyta [19] & $72.2 \%$ & Kato-katz and Formol-ether & 503 \\
\hline & Jimma Zone [20] & $48.4 \%$ & Kato-katz & 302 \\
\hline
\end{tabular}

Helminth infections bring multiple health problems with a long term effect of growth retardation, reduced mental development, increased susceptibility to other infections and malnutrition, low academic performance and school absenteeism [5, 22]. Considering the health impact of both STH and schistosomiasis in the country, Ethiopia has launched a national deworming program in November 2015 giving priority to SAC. In the same year, 13 and 5 million SAC were treated for STH and schistosomiais respectively [4].

Arba Minch University, where all authors of the present study are affiliated, has been conducting independent monitoring of the national deworming program since 2015. One of the major challenges we observed was difficulty in accessing and convincing non-enrolled children to take drugs (unpublished data). Despite this, most of the previous deworming monitoring and coverage surveys were conducted in primary schools; excluding non-enrolled children. Moreover, the deworming program is not integrated with water, sanitation and hygiene (WASH) program as well as vector control (for Schistosomiasis) making re-infection common. Factors such as poor environmental sanitation and personal hygiene predispose people for intestinal parasitic infection. In addition, several occupational and socio-cultural factors affect the level of risk at different geographical settings [9, 19-21, 23, 24]. Except in one study [17], predisposing factors for intestinal parasitosis are also not well studied for primary school age children at community level. Hence the aim of the present study was to conduct a community based assessment on the burden of intestinal helminths and associated factors three years after initiation of the national mass drug administration (MDA) in Arbaminch Zuria district, Southern Ethiopia.

\section{Methods}

\section{Study design and area}

A community based cross-sectional study was conducted among SAC (5 to 14 years) living in Health Demographic Surveillance Site (HDSS) of Arbaminch Zuria district, Gamo Gofa Zone, Southern Ethiopia. The district lies surrounding Arbaminch town which is located about $454 \mathrm{~km}$ south of Addis Ababa, the capital of Ethiopia. The district is composed of a total of 31 kebeles (neighbourhood) with three different climatic zones: high land, midland and lowland. The HDSS include 9 kebeles representing all the three climatic zones.

\section{Sample size and sampling technique}

This study is part of a large scale project investigating 'Undernutrition and Its Associated Factors among School Age Children in Arbaminch Zuria District'. Prevalence of undernutrition and perceived factors were considered to calculate the sample size of the project by referring previous findings [25-28]. Hence, 405 SAC were recruited to participate. All 9 kebeles found within the limit of HDSS were selected purposively to easily access a sampling frame of households with SAC. Proportional allocation based on the number of households with SAC was made (Fig. 1). Then, households with SAC were selected by simple random sampling technique. If there is only one eligible SAC in the selected household, he/she was involved in the study; however, if more than one eligible SAC are there, one was selected 


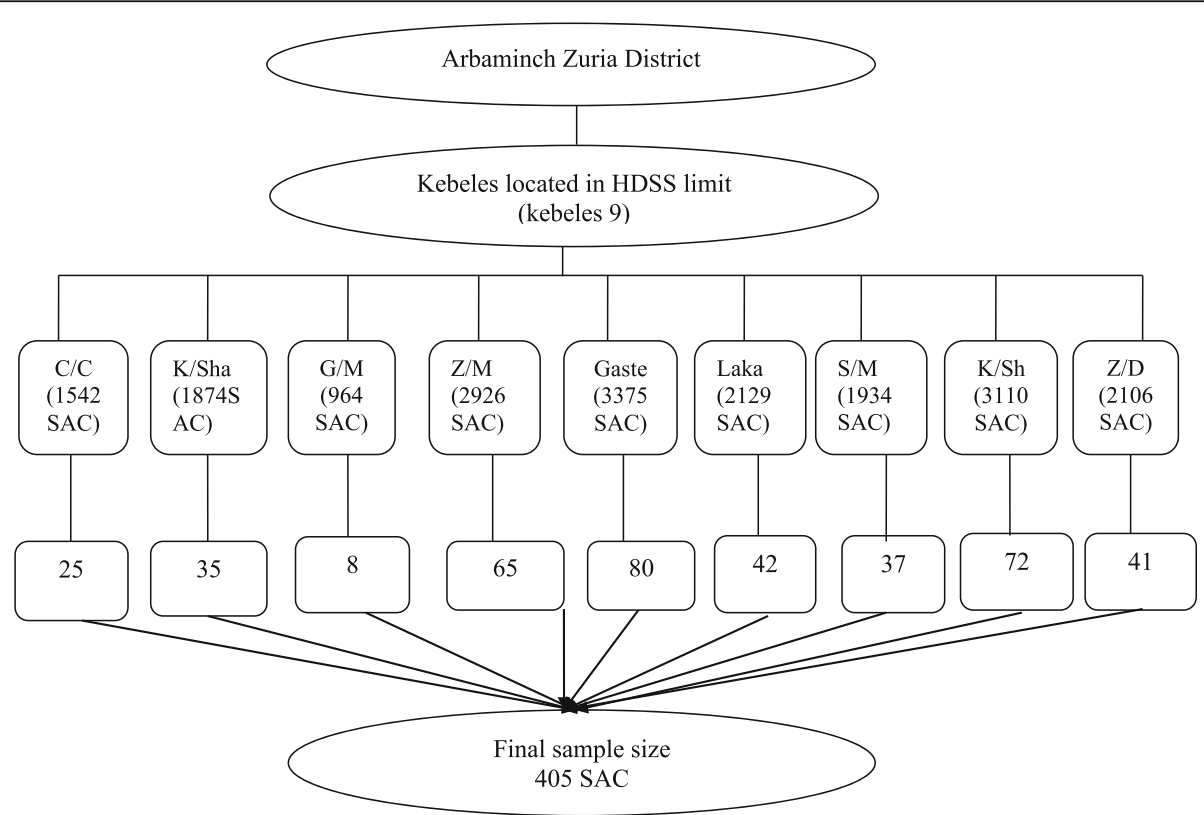

Fig. 1 Schematic presentation of sampling procedure for the study on burden of intestinal helminths and associated factors among school age children in Arbaminch Zuria district, Southern Ethiopia, 2017. (C/C-Chano Chalba, K/Sha- Kola Shara, G/M- Genta Mecha, Z/M- Zigiti Merche, S/MShelle mella, K/Sh- Kolla shelle, Z/D- Zaise Dambille)

for the study by lottery method. Children who were critically ill during the time of data collection and those residing in the study area for less than 6 months were excluded from the study.

\section{Data collection}

\section{Socio-demographic characteristics}

Medical laboratory technicians who are fluent speakers in the local language (Gamo) were trained for data collection. A pretested structured questionnaire was translated to local language and administered through face to face interview to caregivers (preferably mothers). It was used to collect data about socio- demographic characteristics and associated factors for intestinal helminthic infections (Additional file 1).

\section{Stool examination}

Each participating child was consulted about sample collection and supplied with labeled plastic stool cup, toilet paper and applicator stick and was instructed to bring about $5 \mathrm{~g}$ of stool. The collected sample was emulsified in $10 \%$ formal water and transported to the Microbiology and Parasitology laboratory of College of Medicine and Health Sciences, Arba Minch University for microscopic examination. About $1 \mathrm{~g}$ stool sample was concentrated and examined using the formol-ether concentration technique following standard protocol as expressed in our previous publication [29] .

\section{Statistical analysis}

Data was edited, cleaned, entered and analyzed using SPSS version 22.0. Descriptive statistics were calculated to describe the study population characteristics. Bivariate logistic regression was used to assess associations between categorical variables. Multivariate regression model then followed for variables with $p \leq 0.25$ in the bivariate analysis. Association between variables was considered statistically significant only if $p$-value $\leq 0.05$ at $95 \%$ confidence level.

\section{Results}

Socio- demographic characteristics of study participants Among 405 SAC recruited, 6 refuse to participate and 8 were unable to produce adequate amount of stool for parasitological examination. Hence data from 391 SAC were complete for analysis. One hundred ninety six (50.1\%) were male and 195 (49.9\%) were female children. The mean age of study participants was 10.1 years old with standard deviation of 2.6 (Table 2).

\section{Prevalence of intestinal Helminths}

One hundred eighty two children (46.5\%; 95\% CI: 41.951.4) were infected with at least one intestinal parasite. Giardia lamblia cyst was the only intestinal protozoa detected; all other infections were caused by helminths. In this study, we will focus on prevalence of helminths (excluding protozoa) which was $46.3 \%$ (95\% CI: 41.9-51.5). In total seven helminths were detected among which $A$. 
Table 2 Socio-demographic characteristics of school age children $(n=391)$ in Arbaminch Zuria district, Southern Ethiopia, 2017

\begin{tabular}{|c|c|c|}
\hline Variables & Category & Frequency (\%) \\
\hline \multirow[t]{2}{*}{ Age Group (In Years) } & $5-11$ & $254(65.0)$ \\
\hline & $12-14$ & $137(35.0)$ \\
\hline \multirow[t]{2}{*}{ Child Sex } & Male & $196(50.1)$ \\
\hline & Female & $195(49.9)$ \\
\hline \multirow[t]{2}{*}{ School Enrolment } & Enrolled & $223(57)$ \\
\hline & Non enrolled & $168(43)$ \\
\hline \multirow[t]{2}{*}{ Grade Level } & $1-4$ class & $169(75.8)$ \\
\hline & $5-8$ class & $54(24.2)$ \\
\hline \multirow[t]{2}{*}{ Birth Order } & $\leq 2$ & $200(51.2)$ \\
\hline & $>2$ & $191(48.8)$ \\
\hline \multirow[t]{3}{*}{ Religion Of Parent/ Care Giver } & Protestant & $241(61.6)$ \\
\hline & Orthodox & $142(36.3)$ \\
\hline & Others & $8(2.0)$ \\
\hline \multirow[t]{4}{*}{ Ethnicity Of Parent/ Care Giver } & Gamo & $338(86.4)$ \\
\hline & Zeise & $35(9.0)$ \\
\hline & Wolaita & $11(2.8)$ \\
\hline & Others & $7(1.8)$ \\
\hline \multirow[t]{4}{*}{ Educational Status Of mother } & No formal education & $257(65.7)$ \\
\hline & Unable to read and write & $38(9.7)$ \\
\hline & Primary level & $83(21.2)$ \\
\hline & Secondary level and above & $13(3.3)$ \\
\hline \multirow[t]{4}{*}{ Occupation Of Mother/ Care Giver } & Housewife & $370(94.6)$ \\
\hline & Government employee & $1(0.3)$ \\
\hline & Private employee & $14(3.6)$ \\
\hline & Others & $6(1.5)$ \\
\hline \multirow[t]{2}{*}{ Family Size } & $<4$ & $18(4.6)$ \\
\hline & $\geq 4$ & $373(95.4)$ \\
\hline \multirow[t]{3}{*}{ Climatic Zone In Which The Household Is Located } & Lowland & $163(41.7)$ \\
\hline & Midland & $44(11.3)$ \\
\hline & Highland & $184(47.1)$ \\
\hline \multirow[t]{2}{*}{ Illness in the last 2 weeks } & Yes & $16(4.1)$ \\
\hline & No & $375(95.9)$ \\
\hline \multirow[t]{2}{*}{ Habit of eating row fruits and vegetables } & Yes & $208(53.2)$ \\
\hline & No & $183(46.8)$ \\
\hline \multirow[t]{2}{*}{ wash fruits and vegetables before consumption } & Yes & $113(54.3)$ \\
\hline & No & $95(45.7)$ \\
\hline \multirow[t]{2}{*}{ Habit of swimming } & Yes & $108(27.6)$ \\
\hline & No & $283(72.4)$ \\
\hline \multirow[t]{2}{*}{ Habit of hand washing after contact with soil } & Yes & $229(58.6)$ \\
\hline & No & $162(41.4)$ \\
\hline \multirow[t]{2}{*}{ Habit of hand washing before meal } & Yes & $277(70.8)$ \\
\hline & No & $114(29.2)$ \\
\hline \multirow[t]{2}{*}{ Habit of hand washing after toilet } & Yes & $265(67.8)$ \\
\hline & No & $126(32.2)$ \\
\hline
\end{tabular}


Table 2 Socio-demographic characteristics of school age children $(n=391)$ in Arbaminch Zuria district, Southern Ethiopia, 2017 (Continued)

\begin{tabular}{|c|c|c|}
\hline Variables & Category & Frequency (\%) \\
\hline \multirow[t]{2}{*}{ Habit of wearing shoe } & Yes & $362(92.5)$ \\
\hline & No & $29(7.5)$ \\
\hline \multirow[t]{2}{*}{ Presence of domestic animals at home } & Yes & $338(86.4)$ \\
\hline & No & $53(13.6)$ \\
\hline \multirow[t]{2}{*}{ Dewormed in last year national deworming campaign } & Yes & $128(32.7)$ \\
\hline & No & $263(67.3)$ \\
\hline \multirow[t]{2}{*}{ Number of times taking deworming in the last year } & Once & $102(79.7)$ \\
\hline & Twice & $26(20.3)$ \\
\hline \multirow[t]{2}{*}{ Latrine availability } & Yes & $374(95.7)$ \\
\hline & No & $17(4.3)$ \\
\hline \multirow[t]{4}{*}{ Source of drinking water } & Pipe water & $303(77.5)$ \\
\hline & Protected well/ spring & $36(9.2)$ \\
\hline & Unprotected well/ spring & $37(9.5)$ \\
\hline & River & $15(3.8)$ \\
\hline
\end{tabular}

lumbricoides was with the highest frequency $(56,14.3 \%)$ followed by hookworms $(55,14.1 \%)$ and Hymenolepis nana $(17,4.3 \%)$ (Table 3).

\section{Factors associated with intestinal Helminth infection}

The bivariate analysis show that prevalence of intestinal helminths was higher in children with age group of 6-11 (48.8\%) years old as compared to $12-14$ (42.3\%) years old children; however the difference was not statistically significant $(p=0.221)$. Latrine availability was strongly associated with intestinal parasitosis according to the

Table 3 Prevalence of intestinal parasites among school age children $(n=391)$ in Arbaminch Zuria district, Southern Ethiopia, 2017

\begin{tabular}{llll}
\hline Parasites & & Frequency \\
\cline { 3 - 4 } & & Number & Percentage \\
\hline Helminths & Ascaris lumbricoides & 56 & 14.3 \\
& Hookworms & 55 & 14.1 \\
& Hymenolepis nana & 17 & 4.3 \\
& Schistosoma mansoni & 12 & 3.1 \\
& Trichuris trichuria & 10 & 2.6 \\
& Taenia species & 4 & 1.0 \\
Protozoa & Entrobius vermicularis & 3 & 0.8 \\
Number of helminth & Giardia lamblia cyst & 1 & 0.3 \\
Species per child & 1 & 158 & 40.4 \\
Total Prevalence & Helminth & 24 & 6.1 \\
& Protozoa & 181 & 46.3 \\
& Helminth + Protozoa & 182 & 46.5 \\
\hline
\end{tabular}

bivariate analysis result $(\mathrm{COR}=1.25 ; 95 \% \mathrm{CI}$ : $1.08-1.79$, $p=0.018$ ) but that was not the case when corrected for confounders in the multivariate analysis $(\mathrm{AOR}=1.43$; 95\%CI: $0.10-1.95, p=0.275)$. In the multivariate regression model, not washing fruits and vegetables before consumption $(\mathrm{AOR}=2.16 ; 95 \% \mathrm{CI}: 1.10-4.25, p=0.026)$ and habit of swimming $(\mathrm{AOR}=1.17 ; 95 \% \mathrm{CI}$ : $1.03-1.95$, $p=0.045)$ were significantly associated with intestinal parasitic infection (Table 4).

\section{Discussion}

Prevalence of intestinal helminth infection in the present study was comparable with $44.05 \%$ prevalence in Kenya, $44.2 \%$ in Tilili and $48.4 \%$ in Jima zone [12, 20, 23]. Lower prevalences ranging from 24.7 to $39.9 \%$ were reported from three separate studies (Tepi, Bale, Chencha,) in Ethiopia [16, 21, 24]. Inclusion of non-enrolled children in the present study might increase the rate of infection because most of non-enrolled children were not dewormed in the previous three rounds of national deworming (unpublished data). Intestinal helminths are more prevalent in areas with hot and humid climate accompanied with poor sanitation. Both Chencha and Bale are highlands with very cold weather condition which delays the transmission of intestinal helminths.

The same study conducted in $2010 / 11$ by Wogayehu et al. shows a prevalence of $39.9 \%$ in Arba Minch Zuria district [17]. After 3 years of bi-annual deworming, we reported increased prevalence in the area (46.5\%). Re-infection due to ineffective implementation of WASH and low deworming coverage for non-enrolled children could justify for the increased prevalence of intestinal helminths. Variations in data collection time 
Table 4 Factors associated with intestinal parasitic infection among school age children $(n=391)$ in Arbaminch Zuria district, Southern Ethiopia, 2017

\begin{tabular}{|c|c|c|c|c|c|c|c|}
\hline Variables & Category & $\begin{array}{l}\text { Number } \\
\text { examined }\end{array}$ & $\begin{array}{l}\text { Rate of helminth } \\
\text { infection N (\%) }\end{array}$ & $\begin{array}{l}\text { COR } \\
(95 \% \mathrm{Cl})\end{array}$ & $\begin{array}{l}p- \\
\text { value }\end{array}$ & $\begin{array}{l}\text { AOR } \\
(95 \% \mathrm{Cl})\end{array}$ & $\begin{array}{l}p- \\
\text { value }\end{array}$ \\
\hline \multirow[t]{2}{*}{ Age group (in years) } & $6-11$ & 254 & $124(48.8)$ & $\begin{array}{l}0.77(0.51- \\
1.17)\end{array}$ & 0.221 & $\begin{array}{l}0.59(0.32- \\
1.11)\end{array}$ & 0.103 \\
\hline & $12-14$ & 137 & $58(42.3)$ & 1 & & 1 & \\
\hline \multirow[t]{2}{*}{ Child sex } & Male & 196 & $90(45.9)$ & 1 & & & \\
\hline & Female & 195 & $92(47.2)$ & $\begin{array}{l}0.95(0.64- \\
1.41)\end{array}$ & 0.803 & & \\
\hline \multirow[t]{2}{*}{ School Enrolment } & Enrolled & 223 & 109(48.9) & 1 & & & \\
\hline & $\begin{array}{l}\text { Non } \\
\text { enrolled }\end{array}$ & 168 & $73(43.5)$ & $\begin{array}{l}1.24(0.83- \\
1.86)\end{array}$ & 0.287 & & \\
\hline \multirow[t]{2}{*}{ Wash fruits and vegetables before consumption } & Yes & 113 & $63(55.8)$ & 1 & 1 & & \\
\hline & No & 95 & $44(46.3)$ & $\begin{array}{l}1.46(0.84- \\
2.53\end{array}$ & 0.176 & $\begin{array}{l}2.16(1.10- \\
4.25)\end{array}$ & 0.026 \\
\hline \multirow[t]{2}{*}{ Habit of swimming } & Yes & 108 & $61(56.5)$ & $\begin{array}{l}1.15(1.03- \\
1.69)\end{array}$ & 0.015 & $\begin{array}{l}1.17(1.03- \\
1.95)\end{array}$ & 0.045 \\
\hline & No & 283 & $121(42.8)$ & 1 & & 1 & \\
\hline \multirow[t]{2}{*}{ Habit of hand washing after toilet } & Yes & 265 & $118(44.5)$ & 1 & & 1 & \\
\hline & No & 126 & $64(50.8)$ & $\begin{array}{l}0.78(0.51- \\
1.19)\end{array}$ & 0.246 & $\begin{array}{l}0.73(0.35- \\
1.54)\end{array}$ & 0.408 \\
\hline \multirow[t]{2}{*}{ Hand washing habit before meal } & Yes & 308 & $130(46.9)$ & 1 & & 1 & \\
\hline & No & 114 & $52(45.6)$ & $\begin{array}{l}1.05(0.68- \\
1.63)\end{array}$ & 0.812 & $\begin{array}{l}0.74(0.31- \\
1.75)\end{array}$ & 0.493 \\
\hline \multirow[t]{2}{*}{ Habit of wearing shoe } & Yes & 362 & $167(46.1)$ & 1 & & & \\
\hline & No & 29 & $15(51.7)$ & $\begin{array}{l}0.97(0.44- \\
2.13)\end{array}$ & 0.933 & & \\
\hline \multirow[t]{2}{*}{ Presence of tame animals at home } & Yes & 338 & $161(47.6)$ & $\begin{array}{l}0.72(0.40- \\
1.30)\end{array}$ & 0.278 & & \\
\hline & No & 53 & 21 (39.6) & 1 & & & \\
\hline \multirow[t]{4}{*}{ Dewormed in last year national deworming campaign } & Yes & 128 & $68(53.1)$ & 1 & & 1 & \\
\hline & No & 263 & $114(43.3)$ & $\begin{array}{l}1.48(0.97- \\
2.26)\end{array}$ & 0.070 & $\begin{array}{l}1.23(0.68- \\
2.22)\end{array}$ & 0.496 \\
\hline & Yes & 374 & $169(45.2)$ & 1 & & 1 & \\
\hline & No & 17 & $13(76.5)$ & $\begin{array}{l}1.25(1.08- \\
1.79)\end{array}$ & 0.018 & $\begin{array}{l}1.43(0.10- \\
1.95)\end{array}$ & 0.275 \\
\hline \multirow[t]{2}{*}{ Source of drinking water } & Safe & 339 & $158(46.6)$ & 1 & & 1 & \\
\hline & Unsafe & 52 & $24(46.2)$ & $\begin{array}{l}1.02(0.57- \\
1.83)\end{array}$ & 0.951 & $\begin{array}{l}1.42(0.54- \\
3.73)\end{array}$ & 0.481 \\
\hline
\end{tabular}

AOR Adjusted Odd Ratio, Cl Confidence Interval, COR Crude Odd Ratio

(season) and sample size also influence magnitude of infection. Wogayehu et al. collected the data for 11 consecutive months (September to July) in order to avoid seasonal variations and the sample size was 858 , which is more representative as compared to the present study [17].

Gawad et al. reported an intestinal parasite infection rate of $90 \%$ in Yemen [6]. Higher prevalences of $64.4 \%$ and $84 \%$ were also reported in two separate studies by Abdulshafi et al... and Siddig et al. respectively from Sudan $[10,11]$. In all the three studies, stool samples were processed for direct wet mount to detect intestinal protozoa; we couldn't do this because we collected data house to house which makes difficult to prepare and examine direct wet mount smear immediately after stool collection.

Gawad et al. performed duplicate Kato Katz technique which increases the sensitivity of detecting helminth ova specially Schistosoma. Findings by Nasiru et al also reveal higher prevalence of intestinal parasitosis (63.49\%) from Nigeria [7]. Inter-country variations in distribution of intestinal parasites and sample size plus method of detection might bring such differences (Table 1). 
Higher prevalences of $65.5 \%$ to $72.2 \%$ were reported in separate studies from Bahir Dar, Maksegnit and Enfranz, Hawassa, Zege and Wolyta [13-15, 18, 19]. Variation in spatial distribution of intestinal helminths, number of study participants recruited and the laboratory techniques applied brought this difference. Moreover, data for the previous studies conducted in Ethiopia were collected before or early after the national deworming program (2015) was implemented in the country $[13,14,18,19]$.

Ascaris lumbricoides was the most frequent intestinal helminth detected. This is in line with the global data denoting that $A$. lumbricoides is the most prevalent helminth in the world with 819 million cases annually [2]. The same is true in the Ethiopian context as supported by previous studies from different parts of the country [16-18, 20, 23]. S. mansoni causes the most common helminthic infection according to studies from Gondar and Wolayta, Ethiopia likely due to variation in geographical distribution of S. mansoni $[15,19]$.

Among all study participants, 24 (6.1\%) acquired two parasites or more. This is much lower as compared to previous findings from Ethiopia. Results from Bahir Dar, Zegie and Hawassa reveal $16.2 \%, 25 \%$ and $25.7 \%$ of the study participants were infected at least with two intestinal parasites respectively $[13,14,18]$. The fact that we didn't detect intestinal protozoa by direct wet mount examination of fresh stool lowers the co-infection rate in the present study. The difference in prevalence of multiple parasitic infections at a time might be varied in relation to level of environmental contamination, level of awareness about parasitic infection and socioeconomic factors [13].

The present study showed that not washing fruits and vegetables before consumption and habit of swimming were strongly associated with the presence of intestinal parasitic infections. Intestinal helminths, whose ova are infective stages, are acquired by ingestion with contaminated food and water as well as oral contact with hands. Except for hookworm and schistosomiasis which penetrates the skin directly, fruits and vegetables eaten in raw are common sources of infection. Hence, SAC who eat fruits and vegetables without washing were 2 times at higher risk of being infected with intestinal parasites as compared to those who washed fruits and vegetables before consumption. Parasitic contamination rates ranging from 22.22 to $57.8 \%$ of fruits and vegetables sold at local markets and at pre-harvest phase have been reported in Ethiopia [30-32]. The present study is in agreement with previous results in Ethiopia [29].

The infective cercaria of schistosoma parasites develop in fresh water dwelling snails. Hence frequent contact with contaminated water poses risk of acquiring schistosomiasis. SAC who frequently swim in rivers and lakes were 1.17 times at higher risk of being infected with intestinal parasites compared to SAC having no habit of swimming. Begna et al. also reported similar finding from Eastern Ethopia that water contact activities predispose for intestinal parasitosis [24]. Hook worms easily infect people who walk barefoot. Hence previous studies show that shoe wearing habit is associated with hook worm infection $[13,23,24]$; but in the present study, the association was not significant. Frequent contact with soil despite shoe wearing and other confounding factors might alter the trend. In previous studies water source, habit of hand washing after toilet and before meal, gender, availability and utilization of latrine were found to be associated with intestinal parasitosis $[13,15,18,20$, $23,24]$. However, all these factors were not associated with intestinal parasitosis in the present study. Hence, large scale study recruiting more number of participants is required in order to drive definitive conclusion.

\section{Conclusion}

Despite 3 years of bi-annual deworming program implementation, intestinal hellminths are still major public health problems in Arba Minch Zuria district. Hence we strongly recommend integration of deworming with WASH and vector control programs in order to control intestinal helminthiasis in Ethiopia and honestly, deworming everyone.

\section{Additional file}

Additional file 1: Questionnaire administered to assess the Burden of intestinal helminths and associated factors 3 years after initiation of Mass drug administration in Arbaminch Zuria district, Southern Ethiopia, 2017. The data contains list of questions asked to children (study participants) in order to collect socio-demographic data and factors associated with intestinal helminth infection. (DOCX $20 \mathrm{~kb}$ )

\section{Abbreviations}

HDSS: Health Demographic Surveillance Site; SAC: School-Age Children; STH: Soil Transmitted Helminths; WASH: Water Hygiene and Sanitation

\section{Acknowledgements \\ We thank Arba Minch HDSS data collectors for their contribution during data collection; and Arba Minch University for funding the research. We are grateful to ethical review board of Arba Minch University for giving ethical clearance. Our regards also go to the study participants and care givers for giving consent to participate in the study.}

\section{Funding}

Fund for data collection and analysis was obtained from Arba Minch University. No fund was obtained for designing of the study, result interpretation and manuscript writing.

\section{Availability of data and materials}

The original data for this study is available from the corresponding author.

\section{Authors' contributions}

EZ conceived and designed the project. EZ, GA and ZA performed the experiment, analyzed the data and wrote the manuscript. All authors read and approved the final manuscript. 


\section{Ethics approval and consent to participate}

Ethical approval for the research was granted by research review board of Arba Minch University with a project code of GOV/AMU/T.H.2.1./CMHS/NUR/ 01/09 and Ethics committee's reference number of CMHS/5203/2010. Permission letter was obtained from Arbaminch Zuria district administrative and health offices. Written consent was obtained from all participating childrens' caregivers. Children, who were infected with intestinal helminths, were de-wormed after communicating with local health offices.

\section{Consent for publication}

Not applicable.

\section{Competing interests}

The authors declare that they have no competing interests.

\section{Publisher's Note}

Springer Nature remains neutral with regard to jurisdictional claims in published maps and institutional affiliations.

\section{Author details}

${ }^{1}$ Department of Medical Laboratory Science, College of Medicine and Health Sciences, Bahir Dar University, Bahir Dar, Ethiopia. ${ }^{2}$ Department of Nursing, College of Medicine and Health Sciences, Arba Minch University, Arba Minch, Ethiopia. ${ }^{3}$ Department of Public Health, College of Medicine and Health Sciences, Arba Minch University, Arba Minch, Ethiopia.

Received: 22 March 2018 Accepted: 15 August 2018

Published online: 29 August 2018

\section{References}

1. World Health Organization. The prevention \& control of Schistosomiasis \& soil transmitted Helminthiasis. Geneva: WHO; 2002

2. Pullan LR, Brooker SJ. The global limits and population at risk of soil transmitted helminth infections in 2010. Parasit Vectors. 2012;5 https://doi. org/10.1186/1756-3305-1185-1181.

3. Finkelstein JL, Schleinitz MD, Carabin H, McGarvey ST. Decision-model estimation of the age-specific disability weight for schistosomiasis japonica: a systematic review of the literature. PLoS Negl Trop Dis. 2008;2(3):e158. https://doi.org/10.1371/journal.pntd.0000158.

4. Ethiopian Federal Ministry of Health: Second Edition of National Neglected Tropical Diseases Master Plan. 2016.

5. Shrestha R, Maharjan M. Prevalence of intestinal helminth parasites among school-children of Bhaktapur district, Nepal. Nepal J Zool. 2013;1(1):48-59.

6. Alwabr GMA, Al-Moayed EE. Prevalence of intestinal parasitic infections among school children of Al-Mahweet governorate, Yemen. Eur J Biol Res. 2016:6(2):64-73.

7. Manir N, Umar LM, Abduhadi BJ. Survey on prevalence of intestinal parasites associated with some primary school aged children in Dutsinma area, Katsina state, Nigeria. MOJ Biol Med. 2017;2(2):00044. https://doi.org/10. 15406/mojbm.2017.02.00044.

8. Liao C-W, Fu C-J, Kao C-Y, Lee Y-L, Chen P-C, Chuang T-W, et al. Prevalence of intestinal parasitic infections among school children in capital areas of the Democratic Republic of São Tomé and Príncipe, West Africa. Afri Health Sci. 2016;16(3):690-7. https://doi.org/10.4314/ahs.v16i3.8.

9. Sun C. Prevalence and associated risk factors of Intestinal Helminths infections among pre-school children (1 to 5 years old) in IDPs settlements of Khartoum state, Sudan. J Glob Health. 2015.

10. Siddig HS, Mohammed IA, Mohammed MN, Bashir AM. Prevalence of intestinal parasites among selected Group of Primary School Children in Alhag Yousif area, Khartoum, Sudan. Int J Med Res Health Sci. 2017;6(8):125-31.

11. Gabbad AA, Elawad MA. Prevalence of intestinal parasite infection in primary school children in Elengaz area, Khartoum, Sudan. Acad Res Int. 2014;5(2):86-90

12. Ngonjo T, Kabiru E, Okoyo C, Kihara J, Andove J, Simiyu E, et al. Current status of soil-transmitted Helminths among school children in Kakamega County, western Kenya. Hindawi J Parasitol Res. 2016; https://doi.org/10. 1155/2016/7680124

13. Hailegebriel T. Prevalence of intestinal parasitic infections and associated risk factors among students at Dona Berber primary school, Bahir Dar, Ethiopia. BMC Infect Dis. 2017;17:362. https://doi.org/10.1186/ s12879-017-2466-X.
14. Abdi M, Nibret E, Munshea A. Prevalence of intestinal helminthic infections and malnutrition among schoolchildren of the Zegie peninsula, northwestern Ethiopia. J Infect Public Health. 2017;10:84-92.

15. Gashaw F, Aemero M, Legesse M, Petros B, Teklehaimanot T, Berhe N, et al. Prevalence of intestinal helminth infection, among school children in Maksegnit and Enfranz towns, northwestern Ethiopia, with emphasis on Schistosoma mansoni infection. Parasit Vectors. 2015;8:567. https://doi.org/ 10.1186/s13071-015-1178-6.

16. Zerdo Z, Yohanes T, Tariku B. Soil-transmitted Helminth reinfection and associated risk factors among school-age children in Chencha District, southern Ethiopia: a cross-sectional study. Hindawi J Parasitol Res. 2016; https://doi.org/10.1155/2016/4737891

17. Wegayehu T, Tsalla T, Seifu B, Teklu T. Prevalence of intestinal parasitic infections among highland and lowland dwellers in Gamo area, South Ethiopia. BMC Public Health. 2013:13:151.

18. Tadege B, Shimelis T. Infections with Schistosoma mansoni and geohelminths among school children dwelling along the shore of the Lake Hawassa, southern Ethiopia. PLoS One. 2017;12(7):e0181547.

19. Alemayehu B, Tomass Z, Wadilo F, Leja D, Liang S, Erko B. Epidemiology of intestinal helminthiasis among school children with emphasis on Schistosoma mansoni infection in Wolaita zone, southern Ethiopia. BMC Public Health. 2017:17:587. https://doi.org/10.1186/s12889-017-4499-x.

20. Tefera E, Belay T, Mekonnen SK, Zeynudin A, Belachew T. Prevalence and intensity of soil transmitted helminths among school children of Mendera elementary school, Jimma, Southwest Ethiopia. Pan Afr Med J. 2017;27:88. https://doi.org/10.11604/pamj.2017.27.88.8817.

21. Belachew EB, Tarko DB, Wallie YM. Prevalence of intestinal helminthic parasitic infections and associated risk factors among students in Tepi town, south West Ethiopia. Sci J Public Health. 2017;5(3):192-7. https://doi.org/10. 11648/j.sjph.20170503.16.

22. Liu C, Luo R, Yi H, Zhang L, Li S, Bai Y, et al. Soil-transmitted helminthes in south western China: a cross-sectional study of links to cognitive ability, nutrition, and school performance among children. PLoS Negl Trop Dis. 2015;9(6):e0003877. https://doi.org/10.1371/journal.pntd.0003877.

23. Abera A, Nibret E. Prevalence of gastrointestinal helminthic infections and associated risk factors among schoolchildren in Tilili town, Northwest Ethiopia. Asian Pac J Trop Med. 2014;7(7):525-30. https://doi.org/10.1016/ S1995-7645(14)60088-2.

24. Tulu B, Taye S, Amsalu E. Prevalence and its associated risk factors of intestinal parasitic infections among Yadot primary school children of south eastern Ethiopia: a cross-sectional study. BMC Res Notes. 2014;7:848. http:// www.biomedcentral.com/1756-0500/7/848

25. Aturupane $H$, Glewwe P, Wisniewski S. The impact of school quality, socioeconomic factors and child health on students' academic performance: evidence from Sri Lankan primary schools. Educ Econ. 2006;21(1):237. https://doi.org/10.1080/09645292.2010.511852.

26. Monarrez-Espino J, Martinez H, Martinez V, Greiner T. Nutritional status of indigenous children at boarding schools in northern Mexico. Eur J Clin Nutr. 2004:58:532-48

27. Mekonnen H, Tadesse T, Kisi T. Malnutrition and its correlates among rural primary school children of Fogera District, Northwest Ethiopia. J Nutr Disord Ther. 2013;S12:002. https://doi.org/10.4172/2161-0509.S12-002.

28. Gutema B, Adissu W, Asress Y, Gedefaw L. Anemia and associated factors among school-age children in Filtu town, Somali region, Southeast Ethiopia. BMC Hematol. 2014;14(13 http://www.biomedcentral. com/2052-1839/14/13):30

29. Alemu G, Mama M. Intestinal helminth co-infection and associated factors among tuberculosis patients in Arba Minch, Ethiopia. BMC Infect Dis. 2017; 17(16) https://doi.org/10.1186/s12879-12017-12195-12871.

30. Benti G, Gemechu F. Parasitic contamination on vegetables irrigated with awash river in selected farms, eastern Showa, Ethiopia. J Parasitol Vector Biol. 2014;5(7):103-9.

31. Tomass Z, Kidane D. Parasitological contamination of wastewater irrigated and raw manure fertilized vegetables in Mekelle city and its suburb, Tigray, Ethiopia. Momona Ethiop J Sci. 2012;4(1):77-99.

32. Tefera T, Biruksew A, Mekonnen Z, Eshetu T. Parasitic contamination of fruits and vegetables collected from selected local Markets of Jimma Town, Southwest Ethiopia. Int Sch Res Not. 2014; https://doi.org/10.1155/2014/ 382715 